\title{
Hot topics from the Assemblies
}

Is it possible to identify exacerbations of mild-to-moderate COPD that do not require antibiotic treatment?

Authors: Miravitlles M, Moragas A, Hernández S, et al.

Chest 2013; DOI: 10.1378/chest.13-0518

Summary: Anthonisen's criteria are often used as a guiding principle in the use of antibiotics for exacerbations of COPD. Using data from 152 patients, the clinical response of the application of Anthonisen's criteria was assessed, along with C-reactive protein levels. Without antibiotics, clinical failure was $19.9 \%$, while amoxicillin/clavulanate application resulted in 9.5\%; however, of the Anthonisen criteria, only an increase in purulent sputum (OR 6.1; $95 \% \mathrm{Cl}$ 1.5-25.0; $p=0.005$ ) was a significant predictor of failure without antibiotics. Elevated CRP concentration (OR 13.4; 95\% Cl 4.6-38.8; $p<0.001$ ) was also shown to be predictive. When combined, Anthonisen criteria and CRP gave an AUC of 0.842 , up from 0.708. Using both the Anthonisen criteria and CRP may help to identify patients with COPD exacerbations safely without the use of antibiotics.

Effects of high altitude and cold air exposure on airway inflammation in patients with asthma

Authors: Seys SF, Daenen M, Dilissen E, et al.

Thorax 2013; DOI: 10.1136/thoraxjnl-2013-203280

Summary: Temperature changes are thought to be triggering factors for asthma, though most patients with well-controlled asthma are able to deal with these external factors. 18 asthmatic patients were assessed before, during and after a trip to the mountain Aconcagua (6965 m). This expedition allowed the testing of hypoxia $\left(\mathrm{FlO}_{2} \mathrm{11}_{1} \%\right)$, cold air exposure $\left(-5^{\circ} \mathrm{C}\right)$, and high altitude on airway inflammation. Spirometry, and markers of inflammation ( $f_{\mathrm{eNO}}$ and induced sputum) were studied. FEV 1 and $f_{\mathrm{eNO}}$ were slightly decreased in normobaric hypoxic conditions before the expedition, and during the expedition FEV 1 and FVC were decreased, while asthma symptoms were slightly increased. After the expedition, sputum neutrophil, myeloperoxidase, IL17 mRNA and VEGF were increased, while Asthma Control Test score and $\mathrm{FEV}_{1}$ were decreased. In conclusion, environmental stress in the form of hypoxia, altitude and cold was associated with a moderate decrease in asthma control, increased airway obstruction and inflammation. Pre-screening cold air exposure had similar results to the expedition recordings suggesting that the air temperature is the most significant contributor to the data.

\section{Perception of chronic respiratory impairment in patients' drawings}

Authors: Luthy C, Cedraschi C, Pasquina P, et al.

J Rehabil Med 2013; DOI: 10.2340/16501977-1179

Summary: Patient communication of their own perception of disease severity is an important aspect in treatment. In a cross-sectional study to explore the perception of dynpnoea in patients with severe COPD, 32 patients were tested using quality-of-life questionnaires and asked to draw their body and represent difficulties in breathing. Hospital Anxiety and Depression Scale showed high scores for depression and anxiety and quality of life was shown as being affected by COPD. The drawings illustrated feelings of tightness with representations of the head and lungs. They provide insight into the nature of patient perception and may help in incorporating patients in rehabilitation programmes.

Telehealth remote monitoring for community-dwelling older adults with chronic obstructive pulmonary disease

Authors: De San Miguel K, Smith J, Lewin G.

TelemedJ E Health 2013; DOI:10.1089/tmj.2012.0244

Summary: The aim of this study was to determine if the ongoing selfmonitoring at home combined with remote telemonitoring by nurses could reduce the incidence of emergency room visits and hospitalisations. In a 6-month randomised controlled trial, participants measured their blood pressure, weight, temperature, pulse and oxygen saturation on a daily basis. The data were transmitted to a nurse for monitoring. The control group receive only instruction. The telehealth group had fewer hospital admissions, shorter length of stay and fewer emergency room visits, though the results were not statistically significant, but annual cost savings were significant at \$2931 per person. Telemonitoring can be beneficial for both the patients and in cost savings for healthcare providers.

Referral to palliative care in COPD and other chronic diseases: a populationbased study

Authors: Beernaert K, Cohen J, Deliens L, et al.

Respir Med 2013; DOI: 10.1016/j.rmed.2013.06.00

Summary: A population-based study was performed to describe how patients with various chronic diseases, including COPD, heart failure, cancer, etc., differ in the frequency and delivery of palliative treatment. Of 2405 deaths, $5 \%$ were from COPD, $4 \%$ from heart failure and $28 \%$ were from cancer. However, patients with COPD were statistically less likely to die than those with heart failure, dementia or cancer, despite the median days from referral to death being $10,12,14$ and 20 , respectively. COPD patients not referred for palliative care were more likely to have curative/life-prolonging treatment than palliative/comforting treatment than the non-referrals in the other groups. Patients with COPD are not given sufficient palliative care compared with other chronic diseases and awareness of the option for palliative care should be increased.

The health economic impact of disease management programs for COPD: a systematic literature review and meta-analysis

Authors: Boland MRS, Tsiachristas A, Kruis AL, et al.

BMC Pulm Med 2013; DOI: 10.1186/1471-2466-13-40

Summary: The cost-effectiveness of COPD disease management programmes is not well established. This study aimed to evaluate the costeffectiveness, as well as the relationship between healthcare costs and health outcomes. 11 studies were included in this literature review. Metaanalysis showed a savings in hospitalisation cost of $€ 1060$ per patient per year and total healthcare utilisation of $€ 898$. Small but positive results were present as a result of disease management programs, but there was great variability dependent on patient, study and intervention characteristics. 